\title{
Commentary
}

\section{An agenda for UK clinical pharmacology Developing and delivering clinical pharmacology in pharmaceutical companies}

\section{Duncan Richards}

\author{
GlaxoSmithKline Academic Discovery Performance Unit, GSK R\&D Addenbrookes Hospital, Hills Road, Cambridge CB2 2GG, UK
}

The challenges of developing new medicines are well known. Effective application of clinical pharmacology expertise is vital to the successful evaluation of potential new medicines. In drug development, this depends on effective integration of diverse skills. Many of these are currently in short supply, but through innovative partnerships between industry and academia there is an opportunity to reinvigorate the discipline by nurturing these key skills to the benefit of both partners. Specific areas of focus should be experimental medicine, modelling and simulation, and translational skills.

\section{The challenge for clinical pharmacology in drug development}

Information derived from clinical pharmacology studies makes up a substantial proportion of the material presented on drug labels and in documents describing product characteristics for the guidance of prescribers (e.g. summaries of product characteristics). If properly prepared, the clinical pharmacology package can provide critical data that differentiates a product from its competitors and guides its safe use in varied and complex patient populations. In order to do this, the influence of clinical pharmacology needs to be felt at the very earliest stages of drug development. As new targets are identified, careful consideration needs to be given to the means by which that target can be engaged. What will the effects be of engaging the target elsewhere in the body? How does this influence the route of delivery, and hence the ideal molecular characteristics of the drug? As more information emerges, the likely effects of off-target actions need to be considered. All of this needs to be set in the context of the patients who are likely to receive the drug.

Clinical pharmacology in clinical development is all about understanding the relation between the desired and undesired actions of a drug (Figure 1). When understanding this is put at the heart of drug development, clinical pharmacology information can be used to drive and guide the whole process. All too often there is a perception that the early stage of development can be achieved with limited evaluation of dose responsiveness and that the rest can be bolted on at the late stages of development in preparation for licensing. This is not only short sighted but can be disastrous for the development programme. There are several examples of drugs for which challenges in defining the clinical pharmacology early in clinical development has led to a substantial difference between the doses explored in phase II efficacy studies and those ultimately used in the clinic (e.g. montelukast, captopril). Clinical pharmacology expertise is therefore something that needs to be integrated into development programmes from early development through to postmarketing. Given that this can take $10-20$ years, providing continuity can be difficult.

As appropriate polypharmacy becomes more widespread, the development clinical pharmacologist has to meet a new range of challenges. Most patients will be elderly, and the pharmacokinetic and pharmacodynamic effects of a compound may be very different from those in the archetypical $70 \mathrm{~kg}$ adult. Traditional approaches, such as single-dose pharmacokinetic interaction studies, provide some information, but conducting a study for each possible drug combination is impractical. Furthermore, these types of studies may be inadequate, because the real impact of co-administration may come from pharmacodynamic interactions that are not typically studied in early development. The clinical pharmacologist therefore needs to be able to integrate a deep understanding of the pharmacokinetic and pharmacodynamic characteristics of a drug with knowledge of the pathophysiology of the disease and how it is treated. 


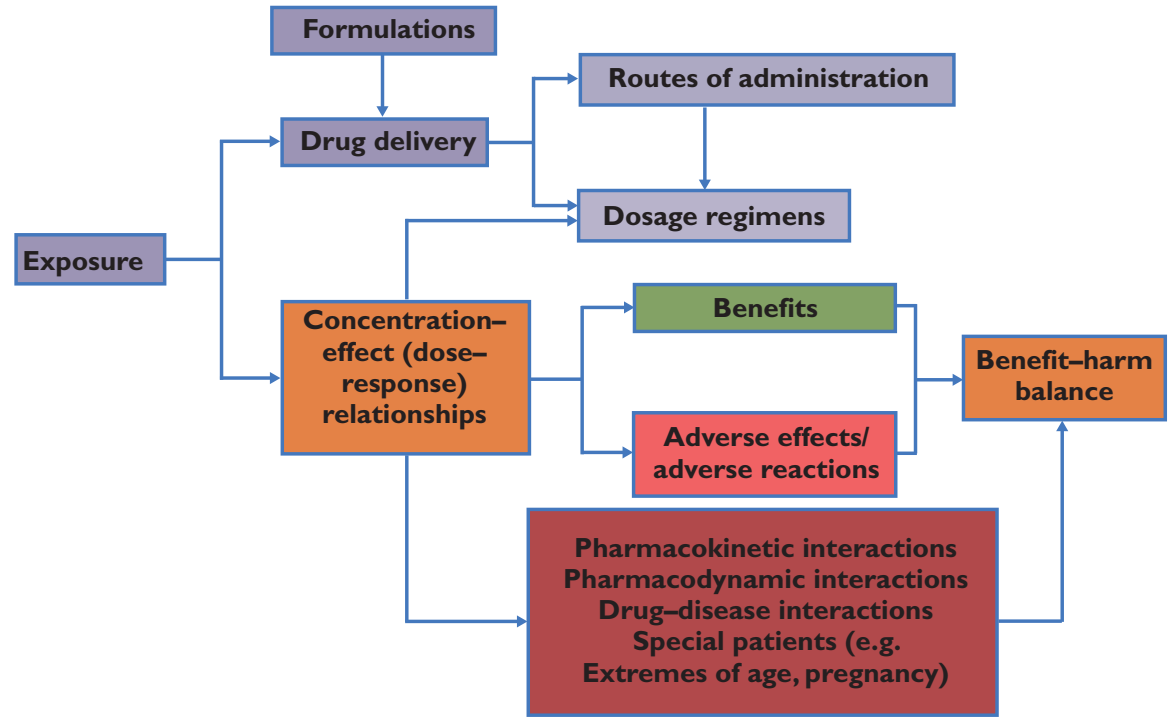

\section{Figure 1}

The roles of clinical pharmacology in drug development: understanding the relation between exposure to a drug and its clinical effects

\section{Delivering clinical pharmacology in pharmaceutical companies}

The skills required by clinical pharmacologists in drug development require integration of a variety of different types of information, diverse but complementary (Figure 2). For example, modelling and simulation are now key features of most drug development programmes $[1,2]$. This relatively new approach has evolved from clinical pharmacokinetics and provides a much more integrated approach to understanding pharmacokinetic-pharmacodynamic relationships. Successful model development requires a particular mix of mathematical and biological skills, contextualized by a thorough understanding of the relevant pathophysiology [3].

The traditional view has been that the clinical pharmacologist is a clinician scientist. However, it is very unusual for an individual to have all the necessary skills, and medically qualified clinical pharmacologists are in short supply. As with all other aspects of drug development, effective delivery of clinical pharmacology information depends on contributions from several team members with specific expertise. There is a particular requirement for the leaders of these teams to be able to integrate these contributions in order to derive a coherent and scientifically robust plan. This requires leadership as well as scientific skills. These skills are not part of a traditional scientific training and need to be taught and developed if teams are to be effective. The most effective leader is the one able to get the best from the team and may not be the most experienced or senior.

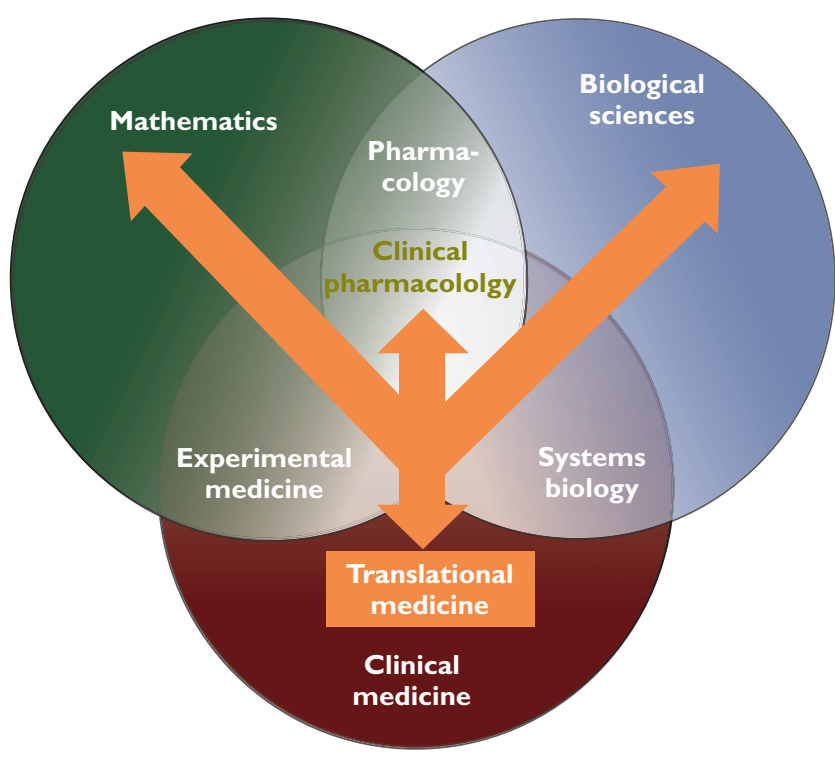

Figure 2

Core skills required to deliver clinical pharmacology in drug development (courtesy of Daren Austin of GlaxoSmithKline)

\section{Developing clinical pharmacology - what are a company's needs?}

Pharmaceutical companies are facing an unprecedented fall in productivity, and the success rate of drug development at phase II may have fallen to as low as 18\% [4]. At the same time, there has been an explosion in our understanding of biology. The gap lies in the translation of this 
knowledge into successful therapies. Following a negative (phase II) study, the question is always, 'was it the molecule or the mechanism?' Increasing the proportion of positive phase II studies is a critical target, but there will always be failures. The right mix of clinical pharmacology skills is vital to improving the success rate, but also to being clear about the reasons for failure, thus avoiding commitment of scarce resources to the same question again and again. All too often, some of the necessary skills are missing from the team. Here I shall identify where skills gaps exist and propose some solutions.

\section{Medicine and experimental medicine}

As a clinical specialty in the UK, clinical pharmacology is one of the very few disciplines to have shrunk over the past decade. A similar pattern has been observed in other countries. At the same time, large numbers of medical students and junior doctors still profess to be interested in therapeutics. The barrier appears to be at that critical specialty training decision point. There is a perceived lack of a clear career path post-training; relatively few trainees secure jobs in Clinical Pharmacology and Therapeutics. To attract the brightest and best to train in clinical pharmacology, there must be a career structure in place that offers potential applicants a clear line of sight to the types of post that they recognize as rewarding. The NHS has consistently failed to nurture clinical pharmacology in the mainstream, and so the scope for that path is limited. A few academic posts for researchers with an interest in translational medicine have been created, and those who have clinical pharmacology skills are well placed to make these a success. Given that most translational medicine takes place in industry, experience in this environment would be valuable training for these posts. However, many trainees are reluctant to take a permanent position in a pharmaceutical company, as this is perceived as a one-way move; but there are many examples of pharmacologists who have moved from industry to academia, Sir James Black and Sir John Vane being the most notable. One potential solution would be the creation of fixed-term secondments to industry (Figure 3). These would be of sufficient duration (1-2 years) to allow those who have been seconded to make useful contributions to projects, while offering an opportunity to return to an academic post at the end of the term. The Wellcome Trust's Translational Medicine and Therapeutics (TMAT) programmes, which are partly supported by pharmaceutical companies, also offer opportunities for academics to develop skills in pharmacology and clinical pharmacology [5].

Experimental medicine suffers from a lack of a common definition, but in this context it is the conduct of a focused clinical experiment in a well-characterized patient group in order to answer a therapeutic question. Clearly, this is closely related to clinical pharmacology, but it may address some aspect of pathophysiology and may not involve a specific therapeutic agent. The UK has a strong tradition in

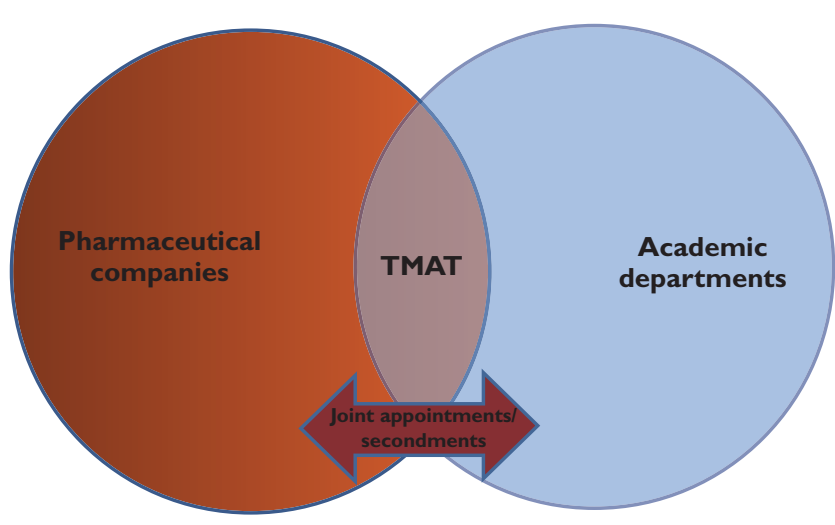

Figure 3

Liaising between industry and academia. Abbreviation: TMAT, translational medicine and therapeutics

experimental medicine, but the current regulatory environment is so all-encompassing and onerous that there is a real danger that work of this sort will no longer be conducted here. Clinical experiments are the lifeblood of experimental medicine and clinical pharmacology, and without a vibrant environment for human clinical experimentation it will be impossible for clinicians to develop the skills needed for new drug research. The report of the Academy of Medical Sciences, which highlights the barriers, is a welcome contribution [6]. The Government's response, if implemented, could go some way to addressing the key issues raised in the report, but it is vital that these are translated into firm actions in the near future [7].

\section{Pharmacology}

Many current drug targets have emerged from advances in our understanding of molecular biology. Many of the innovators of these new potential medicines do not consider themselves pharmacologists, although pharmacological principles are vital to the characterization of a drug target. Learned societies, such as the British Pharmacological Society, have an important role to play in promoting the discipline beyond traditional university departments of pharmacology and clinical pharmacology.

\section{Biology}

Most current drug targets are pluripotent, and while in vitro techniques are important, appropriate in vivo animal models provide an important way to begin to understand potential effects in man. A gap in in vivo skills was identified several years ago, and in response, four projects to build capacity in integrative mammalian biology have been created [8]. Supported by a consortium from Government, academia and industry, this type of programme is essential to ensure that the UK continues to train young 
scientists who are capable of high-quality experimental design and execution, while maintaining the highest standards of animal welfare.

\section{Mathematics}

For a long time, the interface between clinical pharmacology and mathematics focused on pharmacokinetic description and modelling. This remains very important, but modelling disease states and the complex pharmacology of many modern drugs requires a wider skill set, including some more familiar in engineering contexts. Furthermore, greater emphasis on pharmacodynamics is needed, particularly in relation to dose responsiveness and physiologically based models. There is an opportunity for universities to look across their departments and build novel and exciting collaborations that could bring different and invigorating approaches to drug development.

\section{Conclusions}

Clinical pharmacology should be at the very heart of drug development throughout the process. Successful delivery of clinical pharmacology information depends on effective integration of diverse skills. Many of these skills are currently in short supply, but through innovative partnerships between industry and academia there is an opportunity to reinvigorate the discipline by nurturing these key skills to the benefit of both partners.

\section{Competing Interests}

Duncan Richards is an employee of GlaxoSmithKline.

\section{REFERENCES}

1 Chien JY, Friedrich S, Heathman MA, de Alwis DP, Sinha V. Pharmacokinetics/pharmacodynamics and the stages of drug development: role of modeling and simulation. AAPS J 2005; 7: E544-59.
2 Jiang W, Kim S, Zhang X, Lionberger RA, Davit BM, Conner DP, Yu LX. The role of predictive biopharmaceutical modeling and simulation in drug development and regulatory evaluation. Int J Pharm 2011; 418: 151-60.

3 Aronson JK. Rational prescribing, appropriate prescribing. $\mathrm{Br} \mathrm{J}$ Clin Pharmacol 2004; 57: 229-30.

4 Arrowsmith J. Trial watch: phase II failures: 2008-2010. Nat Rev Drug Discov 2011; 10: 328-9.

5 Wellcome Trust. Translational Medicine and Therapeutics Programmes. Available at http://www.wellcome.ac.uk/ Funding/Biomedical-science/Funding-schemes/ PhD-funding-and-undergraduate-opportunities/ WTD027975.htm (last accessed 23 November 2011).

6 The Academy of Medical Sciences. A new pathway for the regulation and governance of health research, January 2011. Available at http://www.acmedsci.ac.uk/p47prid88.html (last accessed 23 November 2011).

7 HM Government. The Plan for Growth, March 2011. Available at http://cdn.hm-treasury.gov.uk/2011budget_growth.pdf (last accessed 23 November 2011).

8 BBSRC-MRc. BBSRC- and MRC-funded studentships to support research training in integrative mammalian biology. Available at http://www.bbsrc.ac.uk/funding/studentships/ strategic-skills.aspx (last accessed 23 November 2011).

\section{RECEIVED}

January 2012

\section{ACCEPTED}

30 January 2012

\section{ACCEPTED ARTICLE PUBLISHED ONLINE} 23 February 2012

\section{CORRESPONDENCE}

Dr Duncan Richards, GlaxoSmithKline Academic Discovery Performance Unit, GSK R\&D Addenbrookes Hospital, Hills Road, Cambridge CB2 2GG, UK.

Tel.: 01223296159

Fax: 01223296108

E-mail: duncan.b.richards@gsk.com 